InnOvaciOnes de NegOciOs 17(33): 21-40

(C) 2020 UANL, Impreso en México (ISSN: 2007-1191)

Recepción: 6 Agosto 2019 Aceptación: 9 Enero 2020

\title{
Predicción de factores macroeconómicos mexicanos mediante el índice de precios y cotizaciones (Prediction of Mexican macroeconomic variables using the price quotation index of the Mexican stock exchange)
}

\author{
Claudia Xelja Rodríguez Vargas, Michael Demmler \\ Universidad Autónoma de Querétaro \\ xeljarv@gmail.com; michael.demmler@uaq.mx
}

\begin{abstract}
The research objective of the present paper is to determine if the Price and Quotation Index of the Mexican Stock Exchange (IPC) can predict the behavior of Mexican macroeconomic variables. The analyzed period is from January 2009 to December 2018 and the chosen variables are Global Economic Activity Index, unemployment rate, exchange rate, inflation rate and interest rate. The methodology of the study involves unit root test and Granger Causality. Moreover, the distributed lag model is used to identify the forecasting period of the IPC with respect to the variables mentioned above. The results show that the IPC is able to predict the behavior of Global Indicator of Economic Activity, unemployment rate and exchange rate immediately and with lags of two and three months.
\end{abstract}

Key words: Price and Quotation Index, Macroeconomic Variables, Prediction

JEL: E32, E37, O4

Resumen: El presente trabajo tiene como objetivo de investigación principal determinar las capacidades predictivas del IPC con respecto al comportamiento de las variables macroeconómicas de México tomando en cuenta datos de enero 2009 a diciembre 2018. Las variables elegidas para el estudio son el IGAE, la tasa de desempleo, el tipo de cambio, la inflación y la tasa de interés las cuales serán analizadas por medio de pruebas de raíz unitaria y causalidad de Granger. Por otro lado, se emplea el modelo de rezagos distribuidos para identificar el periodo de tiempo que tarda en reflejarse el comportamiento del IPC en las variables mencionadas. De acuerdo a los resultados, el IPC es capaz de predecir el comportamiento de las variables IGAE, tasa de desempleo y tipo de cambio de manera inmediata y con rezagos de dos y tres meses.

Palabras Clave: Índice de Precios y Cotizaciones, Factores Macroeconómicos, Predicción 


\section{Introducción}

Existen dos líneas de investigación encaminadas a definir la dirección de causalidad entre el mercado de valores y la economía real, la primera considera que el crecimiento económico determina el comportamiento del mercado de valores mientras que la segunda indica que el desarrollo del mercado de valores determina el comportamiento de la economía real (Morales y Rendón, 1997). A pesar de la existencia de diversos estudios, tanto a nivel internacional como nacional, no se ha definido la causalidad, ya que se ha encontrado evidencia empírica que afirman ambas líneas. Para el caso de México se han realizado trabajos que contemplan hasta el año 2015; sin embargo, se analizan variables económicas y bases de datos distintas al presente artículo. Por lo tanto, el presente estudio ofrece un grado de innovación con respecto a variables contempladas y actualidad de datos.

El indicador principal del mercado de valores mexicano es el Índice de Precios y Cotizaciones publicado por la Bolsa Mexicana de Valores, el cual muestra el comportamiento de los precios de las acciones. Por lo anterior, la presente investigación tiene como objetivo principal determinar las capacidades predictivas del Índice de Precios y Cotizaciones con respecto al comportamiento de los factores macroeconómicos: Indicador Global de la Actividad Económica (IGAE), tasa de desempleo, tipo de cambio, tasa de inflación y tasa de interés de México tomando en cuenta datos históricos del periodo comprendido de enero 2009 a diciembre 2018. La hipótesis de investigación planteada es que el IPC es capaz de predecir el comportamiento de los factores macroeconómicos de México.

El presente artículo consta de cinco capítulos, en el primero de ellos se plantean las teorías de la relación existente entre el desarrollo del mercado financiero y el crecimiento económico, específicamente las llamadas supplyleading y demand-leading descritas por Hugh Patrick en 1966. Además, se presentan estudios realizados a nivel internacional y nacional del tema. El siguiente capítulo corresponde a la metodología, la cual consta de pruebas de raíz unitaria y causalidad de Granger, empleadas para determinar en primera instancia si existe causalidad entre el IPC y las variables macroeconómicas. Además, se muestra un modelo de rezagos distribuidos para identificar el periodo de tiempo en que los cambios en el IPC se reflejan en dichas variables. Los resultados se muestran en el tercer capítulo, donde

Rodríguez, C., Demmler, M. 
se analizan los resultados de las pruebas planteadas en la metodología; en el cuarto capítulo se desarrolla la discusión y en el quinto capítulo se presentan las conclusiones.

\section{Relación entre la Economía Real y el Mercado Financiero}

De acuerdo a Smith (1776) los principales factores del crecimiento económico son la división del trabajo, la eficacia de la actividad productiva y la acumulación del capital. Al igual que Smith, Solow (1957) plantea que el crecimiento económico es determinado por la acumulación de capital, además de la productividad del trabajo, inversión en capital humano y progreso técnico, las cuales son producto de la inversión en investigación y desarrollo. El enfoque situacional del sistema financiero fundamenta la relación positiva entre el desarrollo financiero y crecimiento económico, ya que éste menciona que la calidad del funcionamiento del sistema financiero impulsa la acumulación de capital y la innovación tecnológica, elementos indispensables para el crecimiento económico (Peraza, 2010).

Los sistemas financieros surgen cuando se requiere eliminar fricciones del mercado, conocidas como costos de transacción e información, con la finalidad de facilitar la asignación de recursos; cuando se detecta la necesidad de eliminar dichas fricciones se impulsa el surgimiento de intermediarios financieros encargados de realizar la asignación de recursos y disminuir los costos de información y transacción. Una vez eliminados los costos de información y transacción habrá un aumento en la acumulación de capital e innovación tecnológica y por ende en crecimiento económico (Smith, 1776; Schumpeter, 1911; Solow, 1957; Merton y Bodie, 1995). En la Figura 1 se muestra el diagrama de la relación existente entre los mercados financieros y el crecimiento económico.

En 1966 Hugh Patrick plantea la causalidad entre el crecimiento económico y el desarrollo del mercado financiero mediante dos hipótesis; la primera es llamada supply-leading que establece que el desarrollo de instituciones financieras y mercados financieros impulsa el crecimiento económico por medio del aumento de la oferta financiera. En cambio, la hipótesis demand-following indica que el crecimiento económico beneficia al desarrollo de los mercados financieros debido al aumento de la demanda de los servicios financieros (Patrick, 1966).

\section{Predicción de factores macroeconómicos mexicanos}


A partir de este momento se ha iniciado un constante debate por la causalidad existente entre estos dos conceptos; un ejemplo es la investigación de Banerjee y Ghosh (1998), siendo su principal objetivo examinar el funcionamiento de las instituciones financieras tomando en cuenta ambas hipótesis (supply-leading y demand-following). Sus resultados sustentan lo establecido en la hipótesis supply-leading con una relación fuerte entre los desembolsos reales y el funcionamiento de las instituciones mientras que la hipótesis demand-following presentaba una relación débil. Sin embargo, a pesar de existir diversos estudios empíricos no se ha logrado definir con certeza la causalidad entre el crecimiento económico y el mercado financiero (López y Rodríguez, 2010).

Figura 1 Relación entre Mercados Financieros y Crecimiento Económico.

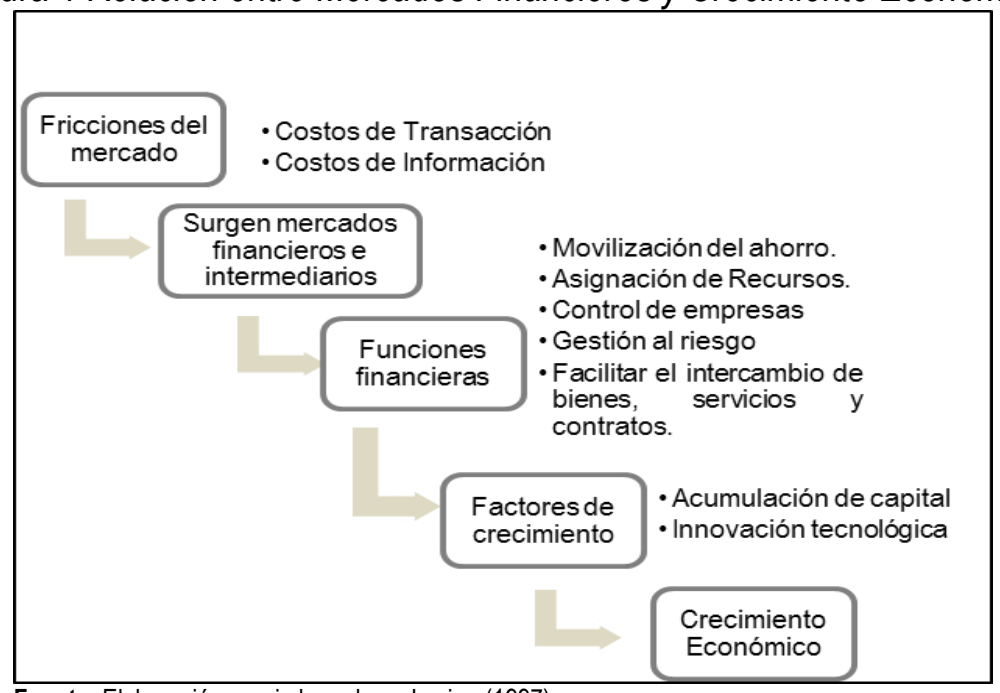

Fuente: Elaboración propia basado en Levine (1997).

Fama (1981) muestra evidencia estadística que sustenta la relación positiva entre los rendimientos del mercado de valores y la actividad económica real, los gastos de capital, la tasa de rendimiento del capital y la producción. Sin embargo, la variable con mayor influencia sobre el rendimiento del mercado de valores corresponde a la tasa de crecimiento de la producción industrial. En el año 1996 los investigadores Habibullah y Baharumshah estudian la relación entre los rendimientos del mercado de

Rodríguez, C., Demmler, M. 
valores y la oferta de divisas, ingreso nacional, nivel de precios del consumo, tasa de interés y tasa de cambio real efectiva en Malasia mediante pruebas de cointegración de Granger. Los datos analizados corresponden al periodo comprendido de enero de 1978 a septiembre de 1992. Los resultados muestran que el nivel de precios del mercado de valores afecta en el nivel de ingresos nominal, el nivel de precios del consumo y la tasa de cambio real, mientras que la oferta monetaria y la tasa de interés afectan el nivel de precios del mercado de valores.

Otro estudio es el realizado por Choi, Hauser y Kopecky (1999), el cual se centra en la relación existente entre los rendimientos rezagados del mercado de valores y la tasa de crecimiento de la producción industrial en Alemania, Canadá, Estados Unidos, Francia, Italia, Japón y Reino Unido. La muestra empleada en este estudio es de enero 1957 a marzo 1996 y fueron analizados con pruebas de causalidad y cointegración. Su estudio concluye que existe una correlación positiva en los datos mensuales entre la tasa de crecimiento de la producción industrial y los rendimientos rezagados del mercado de valores en Reino Unido, Japón y Canadá.

A nivel Latinoamérica existen estudios que analizan la relación entre el desarrollo financiero y el crecimiento económico, tal es el caso del trabajo desarrollado por Ruiz (2004) quien evalúa si el comportamiento de los agentes en los mercados de crédito, bonos y acciones impulsa al crecimiento económico, lo anterior bajo la teoría del crecimiento endógeno. La muestra contempla a Argentina, Brasil, Chile, Colombia y México en el periodo de 1945-1998. Las herramientas econométricas empleadas en el estudio son regresiones de MCO y modelos de SERSA Zellner-Schmidt. Sus resultados determinan que el enfoque de SERSA es más adecuado para realizar dicho análisis debido a las externalidades contempladas en el modelo. Los países donde la inversión corriente influye en el crecimiento económico son Brasil, Chile, Colombia y México; también, sugieren que la interdependencia y las externalidades son elementos importantes tanto a nivel país como regional; por lo tanto, el emplear estrategias en coordinación con otros países podría ser útil para impulsar el crecimiento económico. En conclusión, encuentran evidencia empírica que los mercados financieros juegan un papel importante en el crecimiento económico.

Otro estudio en Latinoamérica, específicamente en Bolivia, es el presentado por Humérez y Yañez (2011) donde analizan la relación existente

\section{Predicción de factores macroeconómicos mexicanos}


entre el desarrollo del sistema financiero y el crecimiento económico. Los datos utilizados son las razones de los agregados monetarios M2 y M3 con respecto al PIB en el periodo comprendido de 2000-2009. Las herramientas utilizadas para el análisis son la estadística y los modelos VAR. Dicho estudio concluye que el $30 \%$ de la tasa de crecimiento es determinada por las variaciones en la tasa de crecimiento del PIB de servicios financieros y profundización. Además, las innovaciones en el sector financiero resultan tener un impacto positivo en la tasa de crecimiento económico. Por lo anterior, su estudio demuestra que el sistema financiero boliviano impulsa el crecimiento económico de Bolivia.

Para el caso de Colombia se encuentra el estudio de Padilla y Zanello (2013) que tiene como objetivo analizar si el desarrollo financiero repercute en el crecimiento económico colombiano. En su estudio se utilizan datos de los 32 departamentos de Colombia en el periodo de 2001-2010, empleando modelos de datos de panel y sección cruzada como metodología. Las variables incluidas en sus modelos son el PIB, el valor del rezago del PIB, colocaciones con respecto al PIB, población, desempleo y tasa de inflación. Los resultados bajo el modelo de sección cruzada muestran que la población y el rezago del PIB no explican el comportamiento del PIB, mientras que la tasa de desempleo tiene un efecto negativo sobre él ya que al aumentar el desempleo en $1 \%$ el PIB disminuye en $0.027 \%$. En cuanto al modelo de datos panel muestra que al aumentar $1 \%$ la variable colocaciones con respecto al PIB, el PIB refleja una disminución del $0.4 \%$. Como conclusión los resultados muestran una relación negativa entre el desarrollo financiero y el crecimiento económico.

En cuanto a estudios en México se encuentra el realizado por Ron (2001) donde analiza la relación de equilibrio y de largo plazo entre la actividad del mercado de valores y el nivel de actividad económica en el periodo de 1987 a 2001. La metodología empleada fue el modelo de cointegración y corrección del equilibrio, los cuales permiten analizar la existencia de relaciones a largo plazo entre variables. Para representar el desarrollo del mercado de valores utiliza tres elementos: tamaño del mercado, su actividad y la liquidez. El tamaño del mercado es representado por el valor del mercado de valores mexicano y el PIB; en cuanto a la liquidez y actividad considera dos indicadores, el primero corresponde al cociente entre el valor total de las operaciones del mercado de valores y el PIB,

\section{Rodríguez, C., Demmler, M.}


explicando la actividad del mercado con respecto al tamaño del mercado; el segundo indicador corresponde a la razón entre la operación total en el mercado de valores y el valor del mercado de valores mexicano para mostrar el grado de actividad del mercado y corroborar el tamaño del mismo. Los indicadores mencionados fueron utilizados para construir el indicador de desarrollo del mercado de valores el cual se integra al modelo junto con las variables macroeconómicas, gasto de gobierno y el tipo de cambio real. Los resultados obtenidos de su estudio muestran que el indicador de desarrollo del mercado de valores tiene una relación positiva con respecto al PIB con un coeficiente de 0.743 . Además del modelo descrito anteriormente determina la relación de equilibrio entre la actividad de la economía real y la actividad bursátil. La variable proxy de la actividad económica real utilizada es el índice de volumen de la producción industrial (VPI) y el IPC es utilizado como representante de la actividad bursátil. Para este modelo se aplicaron pruebas de ADF y PP y la prueba Johansen (modelo de cointegración) para conocer la relación existente a largo plazo y para el equilibrio a largo plazo, dando como resultado, bajo un nivel de confianza del $99 \%$, que los precios del mercado de valores son un predictor de la actividad industrial en México hasta por cinco meses de antelación.

El estudio de Ron (2001) también incluye una prueba de corrección de error mediante la cual se complementa el resultado de relación entre el IPC y el VPI en el corto y largo plazo. Los resultados en el corto plazo muestran que el crecimiento del IPC se refleja de manera positiva en el VPI por cuatro meses; sin embargo, existe una inercia que impulsa al VPI derivada del efecto positivo llevando el efecto de cuatro a doce meses. Por último, Ron (2001) aplica la prueba de causalidad de Granger para cuatro y seis meses de rezagos dando como resultado que el IPC puede predecir el VPI con una antelación máxima de seis meses. Con base en los datos descritos anteriormente el autor concluye que en cuanto mejor sea el desarrollo del mercado de valores mayor será el nivel de la actividad económica en México.

Un estudio más reciente para el mercado mexicano corresponde al de Zavaleta y Martínez (2015) quienes analizan la influencia del desarrollo del mercado de capitales en el crecimiento económico en el periodo del 2000 2015 por medio de un modelo econométrico estructural. Las variables utilizadas en este estudio es el Índice Global de la Actividad Económica (IGAE) como variable proxy del comportamiento del crecimiento económico,

\section{Predicción de factores macroeconómicos mexicanos}


para el desempeño del mercado de capitales de la BMV se utilizó el Promedio Mensual del Índice de Precios y Cotizaciones (IPCP); además, se incluye el nivel de desempleo, el total de las remesas, la Balanza Comercial Petrolera, Inversión Fija Bruta indizada al año 2003, la Balanza Comercial No Petrolera y la Inversión Directa en el Consejo Nacional de Ciencia y Tecnología (CONACYT). Concluye que el IPCP influye en el IGAE en un lapso de 2 meses con un efecto del $0.08 \%$.

Por otro lado, el trabajo de López y Rodríguez (2010), basado en el elaborado por Ron (2001), tiene como objetivo comprobar la existencia de una relación estable y de equilibrio entre el mercado de valores y la actividad económica real en México en el periodo de 1990-2004 mediante análisis de cointegración y modelos de corrección del error. La variable proxy del mercado de valores corresponde al logaritmo natural del IPC y el logaritmo natural del Índice de Volumen de la Producción Industrial (IVPI) como variable representativa de la actividad económica. Sus resultados concluyen que el desarrollo financiero tiene repercusiones positivas en el crecimiento económico encontrando evidencia de causalidad en el sentido de Granger con un nivel de significancia del $10 \%$. Otro resultado se deriva de la aplicación de la estimación MGARCH donde se determina que la volatilidad del IPC influye en la volatilidad del IVPI y viceversa.

La presente investigación se basa parcialmente en los estudios anteriormente mencionados y se eligen las principales variables económicas mexicanas que se definen conceptualmente a continuación. La primera de ellas corresponde al IGAE, calculado mensualmente, el cual representa la generación de riqueza y evolución del sector real de la economía del país siendo una herramienta útil para la toma de decisiones. La información utilizada para el cálculo del IGAE tiene su origen en las encuestas y censos realizados por el INEGI, además de las estadísticas determinadas por diferentes organismos como la Secretaria de Hacienda y Crédito Público (SHCP), Secretaria de Turismo (SECTUR), Banco de México (Banxico), entre otros. La segunda variable es la tasa de desempleo calculada por el INEGI y corresponde al porcentaje de personas que no cuentan con un trabajo pero que están en busca de él con respecto al total de la población que está en edad de trabajar, la cual se considera a partir de los 15 años.

El tipo de cambio, como tercera variable, es una variable macroeconómica importante en la economía mexicana, principalmente la

Rodríguez, C., Demmler, M. 
paridad peso-dólar debido a que la mayoría de las transacciones comerciales internacionales que realiza México es en dólares. Los encargados de la política cambiaria en México es Banxico y SHCP; ambos forman la Comisión de Cambios la cual se encarga del cálculo del tipo de cambio FIX que es un promedio de las cotizaciones realizadas en el mercado cambiario que son liquidables 24 horas después; éste es publicado en el Diario Oficial de la Federación el siguiente día hábil de aquel que corresponde (Banxico, 2018a). La siguiente variable es la inflación y se determina mediante el Índice Nacional de Precios al Consumidor (INPC) el cual se calcula utilizando la fórmula de Laspeyres una vez que se han cotizado los precios de productos de primera necesidad y esparcimiento en 55 lugares en los 32 Estados de la República Mexicana; dichos productos deben de abarcar los sectores agrícola, ganadero, industrial y de servicios. Por último, la variable tasa de interés (TIIE) es calculada por Banxico tomando como base las operaciones que las instituciones de crédito realicen con la intención de que ésta logre representar y transmitir las condiciones actuales del mercado.

\section{Metodología}

La presente investigación es de carácter cuantitativo, explicativo y correlacional ya que a partir de datos de un periodo de tiempo se aplican modelos estadísticos con la intención de explicar la relación existente entre el mercado accionario y el crecimiento económico de México. La base de datos utilizada es mensual y se conforma de 720 datos por cada variable en un periodo de 10 años (2009-2018). El periodo elegido representa una muestra que integra todas las diferentes fases del ciclo económico. Como ya se mencionó en la introducción el objetivo principal del presente trabajo es determinar las capacidades predictivas del Índice de Precios y Cotizaciones con respecto al comportamiento de los factores macroeconómicos: Indicador Global de la Actividad Económica (IGAE), tasa de desempleo, tipo de cambio, tasa de inflación y tasa de interés de México tomando en cuenta datos históricos del periodo comprendido de enero 2009 a diciembre 2018.

Las series de tiempo pueden llegar a manifestar comportamientos que no muestran una relación entre las variables comparadas; sin embargo, no significa que dichas variables no tengan una relación o causalidad. Por ello, es necesario comprobar la existencia de alguna relación entre las variables

\section{Predicción de factores macroeconómicos mexicanos}


del modelo, en este caso se hace uso de la prueba de causalidad de Granger entre el IPC y cada uno de los factores macroeconómicos (Gujarati, 2010; Wooldridge, 2009). Para eliminar la causalidad espuria es necesario conocer si las variables a analizar son estacionarias o no, por lo que se utilizaron las pruebas de raíz unitaria Dickey-Fuller (DF) y Phillips-Perron (PP), las cuales fueron determinadas con la herramienta XLSTAT del programa Excel. Una vez determinada la estacionariedad de las variables se procede a aplicar la prueba de causalidad de Granger con un nivel de significancia del 5\%, para lo que fue necesario establecer un número de rezagos para ambas variables, en este caso se eligieron tres rezagos en cada una de las variables; siendo la regla de decisión: si el $F_{\text {calculado }}$ es mayor al $F_{\text {critico }}$ indica que existe causalidad entre las variables analizadas.

Para identificar el mes en el que se origina la influencia del IPC sobre los factores macroeconómicos se aplicó el modelo de rezagos distribuidos finitos en donde se identifica el impacto rezagado de $z$ en y, a un nivel de significancia del 5\% (Wooldridge, 2009). Para determinar la existencia de influencia en la variable se aplica la regla de decisión: si el valor $p$ es menor al nivel de significancia elegido se dice que dicha variable tiene una influencia sobre la variable y en el tiempo t. La ecuación general de éste modelo está planteada de la siguiente manera:

$$
Y_{t}=\alpha+\delta_{0} z_{t}+\delta_{1} z_{t-1}+\delta_{2} z_{t-2}+\delta_{n} z_{t-n}+u_{t}
$$

Dónde:

$\mathrm{Y}_{\mathrm{t}}=$ Variable dependiente

$\alpha=$ Intersección

$\delta=$ Coeficiente de las variables rezagadas

$\mathrm{z}_{\mathrm{t}}=$ Variable independiente

$\mathrm{Z}_{\mathrm{t}-1}=$ Variable independiente con un rezago

$\mathrm{z}_{\mathrm{t}-2}=$ Variable independiente con dos rezagos

$\mathrm{z}_{\mathrm{t}-\mathrm{n}}=$ Variable independiente con un número de rezagos

indefinidos.

$\mathrm{u}_{\mathrm{t}}=$ error

La variable independiente del modelo corresponde al RMIPC; éste es una muestra representativa del mercado accionario en México ya que integra

Rodríguez, C., Demmler, M. 
a 35 empresas de diferentes sectores con la mayor liquidez y rentabilidad del mercado. Ésta variable es publicada por la BMV, la cual se obtuvo de manera mensual. El periodo a estudiar corresponde de enero 2009 a diciembre 2018. Sin embargo, para el presente estudio fue necesario determinar rendimientos mensuales simples con la intención de obtener datos comparables para el análisis.

Como variables dependientes se eligieron los indicadores macroeconómicos más importantes, los cuales corresponden al IGAE, tasa de desempleo, tipo de cambio, inflación y tasa de interés. La primera de ellas (IGAE) corresponde a la variación porcentual mensual desestacionalizada con respecto al mes anterior del periodo comprendido de enero 2009 a diciembre 2018. La base de datos fue obtenida de la página del INEGI (2018a). Se espera que cuando el RMIPC aumente el IGAE reaccione de la misma manera, pero rezagada. A pesar de que el PIB es una variable significativa que mide el crecimiento y evolución económica de un país fue excluida del modelo debido a su temporalidad ya que al ser determinado de manera trimestral y anual reduce significativamente la base de datos empleada en el estudio. Por lo anterior, se decidió incluir en el modelo al IGAE el cual es calculado mensualmente por el INEGI mediante una metodología similar a la empleada en el cálculo del PIB trimestral. El IGAE ha sido utilizado en estudios similares al presente trabajo como variable proxy del crecimiento económico, un ejemplo de dichos estudios es el realizado por Zavaleta y Martínez (2015).

La segunda variable es la tasa de desempleo (VPMTD) la cual se obtuvo de la página del INEGI (2018b), la base de datos es mensual de enero 2009 a diciembre 2018 y se refleja en porcentaje. Sin embargo, con la intención de ser comparable con la variable independiente se determinó la variación porcentual mensual con respecto al mes anterior. La importancia de incluir dicha variable en el modelo es debido a que ésta es un factor macroeconómico que mide el bienestar de la población de un país ya que al reflejarse una tasa elevada significa una reducción de los recursos financieros con los cuales satisfarán sus necesidades básicas, dando como consecuencia la disminución de la actividad económica y por ende el crecimiento económico (Mingorance, Calva y Barruso, 2017). Se espera que ésta variable tenga un comportamiento inverso con respecto al RMIPC de manera rezagada.

\section{Predicción de factores macroeconómicos mexicanos}


El tipo de cambio (PTC) se obtuvo de la página del Banxico (2018a) del periodo de enero 2009 a diciembre 2018. El tipo de cambio seleccionado para el modelo es el tipo FIX ya que este es calculado por Banxico y es un promedio de las cotizaciones realizadas en el mercado cambiario que son liquidables 24 horas después; además de que éste es generalmente utilizado para el pago de las operaciones en moneda extranjera (Banxico, 2018a); lo cual afecta al comercio internacional elevando los costos de exportación e importación (Ladrón de Guevara y Madrid, 2014). La base de datos obtenida es diaria por lo que fue necesario determinar la variación porcentual diaria y posteriormente calcular un promedio mensual de las variaciones obtenidas. Se espera que el tipo de cambio tenga un comportamiento inverso con respecto al RMIPC, es decir, que cuando el RMIPC presente un aumento el tipo de cambio reflejará una disminución de manera rezagada. La inflación (VPMI) afecta el poder adquisitivo de los consumidores ya que este disminuye al elevarse los precios de las mercancías. De acuerdo al estudio realizado por Andrés y Hernando (1996), la inflación influye negativamente en el crecimiento económico a largo plazo. Por lo anterior, éste indicador se incluye como variable al presente trabajo. La base de datos obtenida corresponde a la variación porcentual mensual y corresponde al periodo de enero 2009 a diciembre 2018, dicha base se obtuvo de la página del INEGI (2018c). Se espera que la inflación presente un aumento cuando el RMIPC muestre un alza o bien disminuya en caso de que el RMIPC presente una baja, lo anterior de manera rezagada.

La última variable corresponde a la tasa de interés (VPMTI), la cual es determinada y controlada por el Banco de México. La tasa de interés es utilizada para estabilizar el tipo de cambio y poder influir en el nivel de la inflación (Levy, s.f); ya que está vinculada con el tipo de cambio e inflación se decide incluirla como variable de la presente investigación. La base de datos corresponde al periodo de enero 2009 a diciembre 2018 y fue obtenida de la página de Banxico (2018b) en porcentaje promedio mensual y posteriormente se calculó la variación porcentual mensual. Al igual que la inflación se espera que ésta variable presente un aumento al aumentar el RMIPC y una disminución cuando el RMIPC caiga, ambos movimientos reflejados de forma rezagada. En la siguiente figura se muestra nuevamente la metodología utilizada.

Rodríguez, C., Demmler, M. 
Figura 2. Metodología empleada

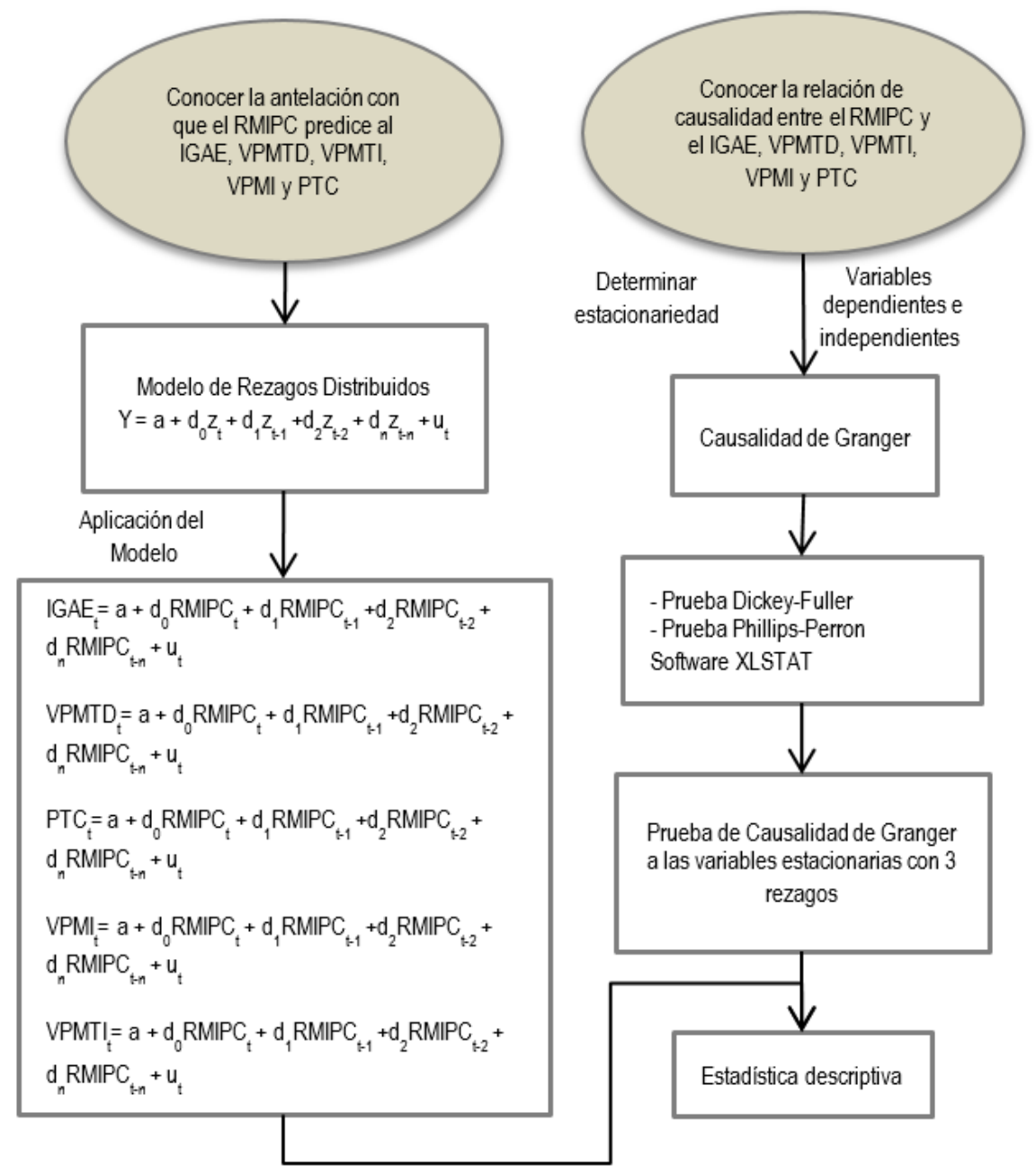

Fuente: Elaboración propia

\section{Resultados}

Los resultados de las pruebas de DF y PP muestran que en la variable independiente (RMIPC) y todas las variables dependientes, IGAE, VPMTD, PTC, VPMI y VPMTI se rechaza la HO, es decir son series estacionarias, lo cual indica que la prueba de causalidad de Granger puede ser aplicada a

\section{Predicción de factores macroeconómicos mexicanos}


cada una de ellas. Los resultados de la prueba de causalidad muestran una relación, a la manera de Granger, del RMIPC hacia las variables utilizadas en el modelo a excepción de la Variación Porcentual Mensual de la Tasa de Interés. Los resultados anteriores se muestran en la Tabla 1 y Tabla 2.

Tabla 1: Prueba Dickey-Fuller (estacionaria)

\begin{tabular}{cccccc}
\hline Variable & $\begin{array}{c}\text { Valor } \mathbf{p} \\
\text { DF }\end{array}$ & $\begin{array}{c}\text { Valor } \mathbf{P} \\
\text { PP }\end{array}$ & $\begin{array}{c}\text { Nivel } \\
\text { Significancia }\end{array}$ & \multicolumn{2}{c}{ Decisión } \\
\hline RMIPC & 0.0002 & 0.0001 & 0.05 & Estacionaria & Estacionaria \\
IGAE & 0.0001 & 0.0001 & 0.05 & Estacionaria & Estacionaria \\
VPMTD & 0.0001 & 0.0001 & 0.05 & Estacionaria & Estacionaria \\
PTC & 0.0002 & 0.0001 & 0.05 & Estacionaria & Estacionaria \\
VPMI & 0.0014 & 0.0001 & 0.05 & Estacionaria & Estacionaria \\
VPMTI & 0.0209 & 0.0001 & 0.05 & Estacionaria & Estacionaria \\
\hline
\end{tabular}

Fuente: Elaboración propia

Tabla 2: Resultados Prueba Causalidad Granger.

\begin{tabular}{llccc}
\hline \multicolumn{2}{c}{ Dirección } & $F_{\text {calculado }}$ & $F_{\text {critico }}$ & Decisión \\
\hline RMIPC $\longrightarrow$ IGAE & 3.1152 & 0.0292 & Causalidad \\
RMIPC $\longrightarrow$ VPMTD & 1.5897 & 0.1960 & Causalidad \\
RMIPC $\longrightarrow$ PTC & 0.8171 & 0.4871 & Causalidad \\
RMIPC $\longrightarrow$ VPMI & 0.7644 & 0.5164 & Causalidad \\
RMIPC $\longrightarrow$ VPMTI & 0.0579 & 0.9800 & No Causalidad \\
\hline
\end{tabular}

Fuente: Elaboración propia

Una vez determinada la causalidad se empleó el modelo de rezagos distribuidos en cada una de las variables. La primera variable correspondiente al IGAE, la cual presenta una influencia positiva rezagada de dos meses con un valor $p$ de 0.0489; para VPMTD se determinó una influencia negativa en el rezago de tres meses con un valor $p$ de 0.0403. En cuanto a la variable de PTC se encontró una influencia negativa inmediata, es decir en el tiempo $t$, con un valor $p$ de 1.43E-06. Por último, las variables VPMTI y VPMI a pesar de haber analizado nueve rezagos no se identifica ninguna influencia sobre ésta.

En la Figura 3 se muestran los coeficientes de los rezagos obtenidos en la regresión de la variable IGAE; el rezago del RMIPC de dos meses tiene

Rodríguez, C., Demmler, M. 
un $\delta$ con valor de 2.654. Para la variable VPMTD sólo se encontró influencia negativa en el rezago de tres meses con un $\delta$ de -0.223 , el cual se muestra en la Figura 4. En cuanto a la variable PTC se encontró influencia negativa inmediata, es decir, tiempo t, con un $\delta$ de -0.010; este valor se muestra en la Figura 5. Cada uno de los valores de $\delta$ mencionadas significan la variación que provoca en la variable dependiente cada unidad de cambio en la variable independiente.

Figura 3. Distribución de IGAE

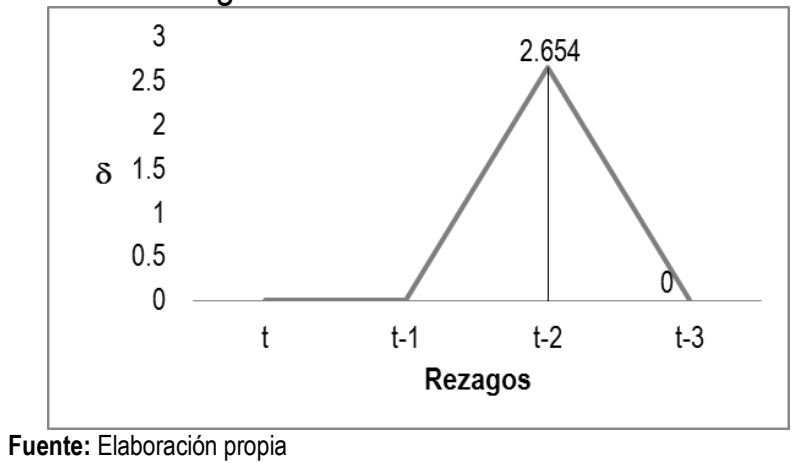

Figura 4. Distribución de rezagos de VPMTD

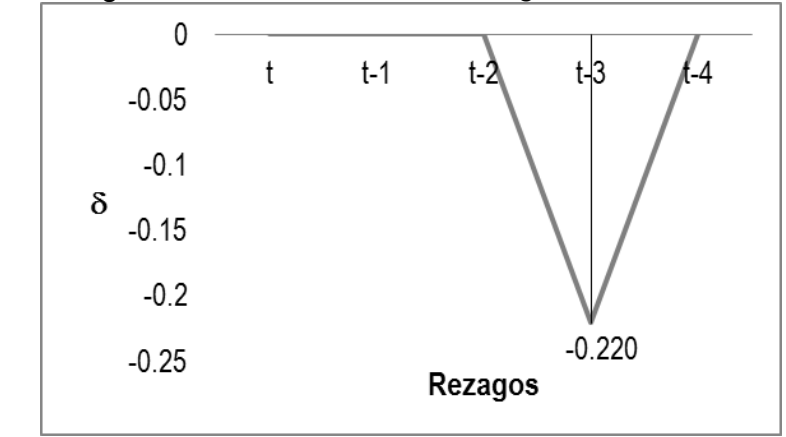

Fuente: Elaboración propia 
Figura 5. Distribución de Rezagos de PTC

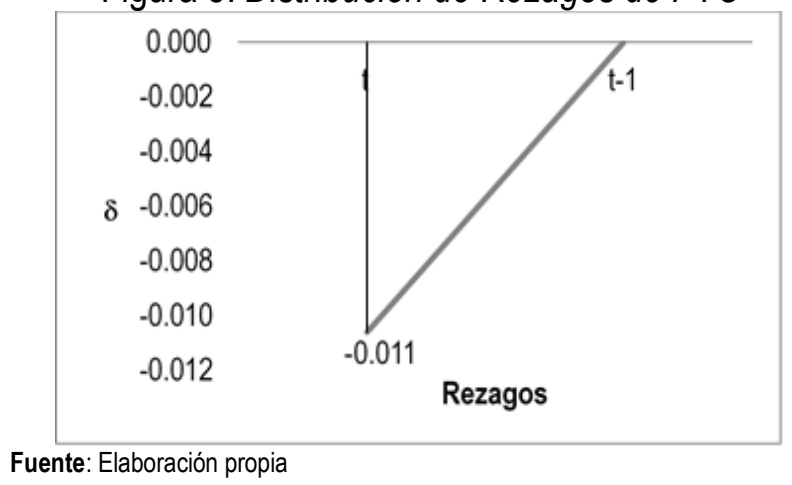

Dentro de la metodología se mencionó el comportamiento esperado de las variables con respecto a un cambio en la variable independiente (RMIPC). Los resultados coinciden con lo esperado a excepción de la VPMI y VPMTI ya que no se obtuvo evidencia estadística de que exista una influencia en forma rezagada. Las variables que muestran una influencia positiva por el RMIPC son el IGAE, la cual es determinada por el signo de la $\delta$ que en este caso es de signo positivo con un valor de 2.6541. Las variables con comportamiento inverso corresponde a las $\delta$ con signo negativo pertenecientes a las variables VPMTD y PTC con un valor de -0.2203 y 0.0106 .

\section{Discusión}

De acuerdo a los resultados presentados anteriormente, se puede observar que el rendimiento del IPC tiene una influencia sobre los factores macroeconómicos (IGAE, tasa de desempleo y tipo de cambio); lo cual arroja evidencia para confirmar la teoría de que el mercado de valores influye y predice el comportamiento de economía real. Lo anterior coincide con los resultados obtenidos en otros trabajos a pesar de la diferencia en la base de datos empleada.

La influencia del IPC sobre el IGAE con dos meses de rezago obtenida en el presente estudio coincide con uno de los resultados del trabajo de Zavaleta y Martínez (2015) quienes comparan estas variables mediante una prueba econométrica estructural; sin embargo, a pesar de que el número de rezagos es el mismo, la variación porcentual es diferente ya que en su

Rodríguez, C., Demmler, M. 
estudio determinan un cambio del $0.08 \%$ en el IGAE por cada punto porcentual del IPC mientras que en el presente estudio se determina un $2.6541 \%$ de cambio en el IGAE por cada punto porcentual del IPC. Se debe considerar que tal diferencia puede derivarse de la base de datos y metodología utilizada en ambos estudios.

Otros estudios que utilizan al IPC como variable representativa del desarrollo del mercado de valores son los realizados por Ron (2001) y López y Rodríguez (2010), ambos estudios comparan el IPC con el volumen de producción industrial; mientras que en el trabajo de Ron (2001) determina un rezago de seis meses entre estas variables el realizado por López y Rodríguez (2010) no arroja pruebas contundentes para afirmar dicha relación. En cuanto al presente estudio coincide con los resultados obtenidos en el trabajo de Ron (2001) en cuanto a la influencia del mercado de valores en la economía mexicana, sin embargo, el tiempo en que tarda en reflejarse difiere con 3 o 4 meses.

Los resultados del presente estudio muestran que las variaciones del rendimiento del IPC influyen en el comportamiento de los factores macroeconómicos correspondientes al IGAE, tasa de desempleo y tipo de cambio durante los primeros tres meses siguientes en que ocurre la variación. La hipótesis de investigación planteada se acepta para dos de las cinco variables seleccionadas en el modelo, IGAE, VPMTD ya que para el PTC aunque existe relación, el cambio es inmediato.

En cuanto a la metodología utilizada en el presente estudio coincide en la aplicación de la prueba de causalidad de Granger con los estudios realizados por Ron (2001) y López y Rodríguez (2010); sin embargo, para determinar el tiempo en que tarda en reflejarse los movimientos del RMIPC se utiliza el modelo de rezagos distribuidos, el cual difiere con los utilizados en los trabajos revisados anteriormente. Además, se analizan variables que en estudios realizados en México no han sido agregadas al trabajo, tal es el ejemplo del tipo de cambio, la tasa de interés y la inflación.

\section{Recomendaciones}

Debido a que los resultados obtenidos confirman la teoría de que el mercado de valores impacta en el crecimiento económico, se considera cumplido el objetivo principal de la presente investigación ya que la

\section{Predicción de factores macroeconómicos mexicanos}


predicción del IGAE y la tasa desempleo con base en el RMIPC en México, resulta útil para prever y aplicar acciones inmediatas encaminadas a disminuir el impacto, en caso de ser negativo, o de impulsarlo, en caso de ser positivo. Lo anterior no sólo beneficia al Gobierno al contar con tiempo para analizar acciones sino también beneficia al sector privado ya que éste al igual que el Gobierno podrá conocer los cambios futuros en la economía y así determinar el impacto que dichos cambios tendrán sobre las empresas, que en caso de ser negativo, afecte lo menos posible su actividad y en caso de ser positivo dará confianza para continuar y aumentar su actividad comercial y de inversión; ambas acciones motor del crecimiento de la economía y bienestar social.

Por lo anterior se considera importante que el Gobierno realice actividades encaminadas al desarrollo y mejoramiento de dicho mercado en México, ya que de esta manera se impulsará el crecimiento económico y por ende el bienestar social. Durante el año 2019 se han aplicado reformas estructurales educativas, de seguridad y económicas importantes por lo que se sugiere realizar en un futuro un estudio que contemple datos de ese año y posteriores para conocer el impacto en la relación existente entre el mercado de valores y las variables macroeconómicas.

Aunque el IPC es el principal indicador de la BMV y aporta información sobre las fluctuaciones generadas en el mercado accionario para conocer el desempeño actual y futuro del mercado, sólo se integra por 35 acciones de mayor liquidez dejando a un lado el resto de las empresas. Al ser integrado por una muestra pequeña, el IPC no logra describir por completo el desempeño del mercado accionario, lo cual representa una limitante en el presente trabajo. Sin embargo, esta variable ha sido utilizada en diferentes estudios como variable representativa del mercado de valores, por ejemplo, Ron (2001), Zavaleta y Martínez (2015), López y Rodríguez (2010).

\section{Referencias}

Andrés, J., y Hernando, I. (1996). ¿Cómo afecta la inflación al crecimiento económico? evidencia para los países de la OCDE. Banco de España: Servicios de Estudios. Recuperado https://www.bde.es/f/webbde/SES/Secciones/Publicaciones/PublicacionesSeriadas/ DocumentosTrabajo/96/Fich/dt9602.pdf

Rodríguez, C., Demmler, M. 
Banco de México (Banxico, 2018a). Mercado Cambiario (Tipos de Cambio). Recuperado de: http://www.anterior.banxico.org.mx/tipcamb/tipCamMIAction.do

Banco de México (Banxico, 2018b). Sistema de Información Económica, Tasa de Interés Interbancaria. Recuperado de: http://www.anterior.banxico.org.mx/Sielnternet/consultarDirectorioInternetAction.do ?sector=18\&accion $=$ consultarCuadro\&idCuadro $=$ CF111\&locale $=e s$

Banerjee, S. S. \& Ghosh, S. (1998). Demand following and supply leading relationships: An empirical analysis. Recuperado de http://mpra.ub.uni-muenchen.de/22443/

Choi, J.; Hauser, S. y Kopecky, K. J. (1999). Does the stock market predict real activity? Time series evidence form the G-7 countries. Journal of Banking \& Finance, 23(12), 1771-1792.

Fama, E. (1981). Stock Returns, Real Activity, Inflation, and Money. The American Economic Review, 71(4), 545-565.

Gujarati, D. y Porter, D. (2010). Econometría (5a ed.). México: Mc Graw Hill.

Habibullah, M. S. y Baharumshah, A. Z. (1996). Money, Output and Stock Prices in Malaysia: An Application of the Cointegration tests. International Economic Journal, 10(2), 121-130.

Humérez, J. y Yañez, E. (2011). Desarrollo del sistema financiero y crecimiento económico: Una aproximación a partir del caso boliviano: 2000-2009. Revista de Análsis, 14, pp. 41-77.

Instituto Nacional de Estadística y Geografía (INEGI, 2018a). Indicador Global de la Actividad Económica. Recuperado en: https://www.inegi.org.mx/temas/igae/

Instituto Nacional de Estadística y Geografía (INEGI, 2018b). Empleo y ocupación. Recuperado en: https://www.inegi.org.mx/temas/empleo/

Instituto Nacional de Estadística y Geografía (INEGI, 2018c). Índice Nacional de Precios al Consumidor. Recuperado en: https://www.inegi.org.mx/programas/inpc/2018/

Ladrón de Guevara, R. y Madrid, R. M. (2014). El tipo de cambio y su efecto en el comercio exterior. Memorias en extenso del XVIII Congreso Internacional de Investigación en Ciencias Administrativas: Admnistración, Gestión de la Innovación y Desarrollo Sustentable, pp. 3935-3963.

Levine, R. (1997). Financial Development and Economic Growth: Views and Agenda. Journal of Economic Literature, 35(2), pp. 688-726.

Levy, N. (s.f). Tasas de interés, demanda efectiva y crecimiento económico. Economíaunam, 9(25), 74-93.

López, F. y Rodríguez, D. (2010). ¿El desarrollo del mercado accionario genera crecimiento económico en México? Un análisis de series de tiempo. Revista Economica, 32, pp. 33-54.

Merton, R. C., y Bodie, Z. (1995). The Global Financial System: A Functional Perspective. En Merton, R. C. y Bodie, Z. A Conceptual Framework for Analyzing the Financial Environment. Boston: Harvard Business School Press.

Mingorance, A. C., Calva, A., y Barruso, B. (2017). Efectos de las variables macroeconómicas e institucionales en el desempleo: Análisis para Europa (19852011). Semestre Económico, 20(42), 17-46.

\section{Predicción de factores macroeconómicos mexicanos}


Morales, A. y Rendón, R. (1997). La Bolsa Mexicana de Valores Realidad económica o especulación? Política y Cultura, 8, pp. 393-410.

Padilla, H. A. y Zanello, L. D. (2013). Relación entre penetración financiera y crecimiento económico. El caso colombiano en el periodo 2001-2010. Revista de economía del Caribe, 11, pp. 35-61.

Patrick H.T. (1966). Financial development and economic growth in under developed countries. Economic Development and Cultural Change, 14, pp. 174-189

Peraza, L. (2010). El papel del mercado bursátil en el crecimiento económico de México. Centro de Estudios de las Finanzas Públicas, pp. 1-60.

Ron, F. E. (2001). Ajuste Dinámico y Equilibrio entre la Producción Industrial y la Actividad Bursátil en México. Momento Económico, 118, pp. 21-38.

Ruiz, A. (2004). Mercados financieros y crecimiento económico en América Latina: Un análisis econométrico. Análisis Económico, 19(40), 141-165.

Schumpeter, J.A. (1911) The Theory of Economic Development. Harvard University Press, Cambridge.

Schumpeter, J. A. (1997). Teoría del Desenvolvimiento Económico: Una investigación sobre ganancias, capital, crédito, interés y ciclo económico. México: Fondo de Cultura Económica.

Smith, A. (1776). An Inquiry into the Nature and Causes of the Wealth of Nations (1a ed.). Londres: W. Strahan \& T. Cadell

Solow, R. M. (1957). Technical Change and the Aggregate Production Function. The Review of Economics and Statistics, 39(3), 312-320.

Wooldrige, F. (2009). Introducción a la econometría. Un enfoque moderno (4a ed.). México: Cengage Learning Editores, S.A. de C.V.

Zavaleta, O. H. y Martínez, I. (2015). Crecimiento económico y desarrollo del mercado de capitales en México. INNOVAR: Revista de Ciencias Administrativas y Sociales, 25, pp. 131-149.

Rodríguez, C., Demmler, M. 